\title{
CALIDAD DE VIDA EN PACIENTES OBESOS SOMETIDOS A SLEEVE GÁSTRICO LAPAROSCÓPICO
}

\author{
Claudia Romero Escobar ${ }^{1 *}$ y Verónica Castro Bocanegra ${ }^{2}$ \\ ${ }^{1,2}$ Corporación Universitaria Rafael Núñez-CURN. Cartagena de indias, Colombia \\ *Autor de correspondencia: claudia.romero@ curn.edu.co
}

Recibido Julio 2018; Aceptado Octubre 2018

\begin{abstract}
Resumen - Se determino la calidad de vida en pacientes sometidos a sleeve gástrico laparoscópico en el E.S.E Hospital Universitario del Caribe en la ciudad de Cartagena de indias. El estudio de tipo descriptivo transversal retrospectivo, se evaluaron 60 pacientes sometidos a este tratamiento desde enero de 2012 a diciembre 2015, a los que se le aplicaron tres cuestionarios: caracterización de las variables socio-demográficos; el sistema de evaluación Score Baros y el cuestionario sobre la calidad de vida Moorehead-Ardelt, incorporado en el Score BAROS. En el estudio el sexo predominante fue el femenino 73,3\%; (n:44), en un 41,7\%; (n=25) de la población estudiadas son empleados, el 38,3\%(n=23) del total de la población son de estrato socio económico 1 , en un 51,7\% $(\mathrm{n}=31)$ el nivel de formación que tuvo más incidencia fue el superior, los datos obtenidos del Score BAROS respecto del grado de éxito de la cirugía bariátrica, se concentró en muy bueno con un $40 \%$, bueno con un $22 \%$ y excelente con un $28 \%$, quedando en los extremos un $3 \%$ de fracaso y un $7 \%$ de regular. Teniendo en cuenta el análisis de los datos obtenidos mediante la aplicación del instrumento, se determinó que el SGL es el tratamiento de elección más efectivo para obtener una mejor calidad de vida luego de que otras medidas terapéuticas hayan fallado.
\end{abstract}

Palabras clave: Obesidad mórbida, cirugía bariátrica, calidad de vida.

\section{QUALITY OF LIFE IN OBESE PATIENTS UNDERGOING LAPAROSCOPIC GASTRIC SLEEVE}

\begin{abstract}
We determined the quality of life in patients undergoing laparoscopic gastric sleeve at the E.S.E. Hospital Universitario del Caribe in the city of Cartagena de Indias. The retrospective cross-sectional descriptive study evaluated 60 patients who underwent this treatment from January 2012 to December 2015. Three questionnaires were applied: characterization of socio-demographic variables; the Baros Score evaluation system and the Moorehead-Ardelt quality of life questionnaire, incorporated in the BAROS Score. In the study the predominant sex was female $73.3 \%$; (n: 44), in a 41.7\% ( $\mathrm{n}=$ $25)$ of the population studied are employees, $38.3 \%(\mathrm{n}=23)$ of the total population are socio-economic stratum 1 , in a $51.7 \%(\mathrm{n}$ $=31$ ) the level of training that had more incidence was higher, the data obtained from the BAROS Score regarding the degree of success of bariatric surgery, was concentrated in very good with $40 \%$, good with $22 \%$ and excellent with $28 \%$, leaving in the extremes a $3 \%$ failure and $7 \%$ regular. Taking into account the analysis of the data obtained through the application of the instrument, it was determined that LMS is the most effective treatment of choice to obtain a better quality of life after other therapeutic measures have failed.
\end{abstract}

Keywords: Morbid obesity, bariatric surgery, quality of life, 


\section{Calidad de vida en pacientes obesos sometidos a sleeve gástrico laparoscópico}

\section{Introducción}

En la actualidad la obesidad constituye un problema de salud pública, dado el importante aumento que ha experimentado su prevalencia en los últimos años y su asociación con enfermedades crónicas no transmisibles como diabetes mellitus tipo 2, hipertensión arterial, dislipidemia, entre otras (Villaba et al., 2017; Basfi-Fer et al., 2012). La Organización Mundial de la Salud (OMS) actualmente la considera como una epidemia global; y constituye la segunda causa evitable de muerte, luego del tabaquismo (Acosta et al., 2010; Marrugo Ligardo, 2017). La alimentación y el estilo de vida sedentario, impactan negativamente en la población, llevándola al desarrollo de sobrepeso. Se han establecido políticas, que buscan cambiar los hábitos alimenticios y fomentar estilos de vida saludable, pero no se ha logrado disminuir la prevalencia de la obesidad (Velásquez-Zambrano et al., 2014).

Cuando los esfuerzos por disminuir la obesidad, sobre todo mórbida, con medidas conservadoras como dietas, ejercicios, medicamentos, no surten efecto, la cirugía bariátrica es una opción. El sleeve gástrico o "manga" gástrica, como tratamiento de la obesidad mórbida (IMC> 40) o sobrepeso, ofrece resultados relacionados con disminución del peso, mejoría de las enfermedades crónicas asociadas, así como menor incidencia de complicaciones tempranas o tardías frente a cualquier otra intervención quirúrgica o procedimiento endoscópico semejante. La pérdida de peso se asocia con mejoría en el estado funcional y el bienestar, pero se desconoce el impacto en la calidad de vida, autoestima, relaciones sexuales, imagen corporal y estado psicológico (Velásquez-Zambrano et al., 2014; Herran Villalba et al., 2016).

La medición de la calidad de vida, permite obtener información sobre una enfermedad determinada y su impacto en la vida del paciente, tal y como él la percibe. Sse emplea en el campo de la cirugía digestiva para valorar la evolución y comprobar la eficacia de tratamientos quirúrgicos. Pero su uso en la cirugía bariátrica no ha sido estandarizado (Pereferrer et al., 2017; Acosta et al., 2010); haciéndose complicada, porque se deben tomar en cuenta diversos aspectos, como patrón la pérdida del exceso de peso, y la mejoría en las condiciones médicas asociadas (Argüello \& Zúñiga, 2016; Acosta et al., 2010).

En respuesta a la necesidad de evaluar de una manera más completa la evolución del paciente luego de cirugía bariátrica, se desarrolló un sistema de análisis que incluye pérdida de peso, mejoría de patologías asociadas y calidad de vida, que se denominó con las siglas BAROS (Bariatric Analysis and Reporting Outcome System), modificado en el 2003, creando el Cuestionario de Calidad de vida, nivel de autoestima y actividad - cuestionario II de Moorehead-Ardelt (Acosta et al., 2010). El propósito fue medir la calidad de vida del paciente obeso luego de haber sido sometido quirúrgicamente a Sleeve Gástrico Laparoscópico (SGL) y de igual manera determinar el grado de éxito de la cirugía, para comprobar que el sleeve gástrico es la mejor opción para mejorar la calidad de vida de estos pacientes.

El objetivo de este estudio fue determinar la calidad de vida en pacientes sometidos a SGL desde el año 2012 al 2015, en la E.S.E Hospital Universitario del Caribe en la ciudad de Cartagena de indias.

\section{Métodos}

Estudio cuantitativo transversal retrospectivo. La población está conformada por los pacientes con obesidad mórbida intervenidos quirúrgicamente a SGL en el E.S.E hospital Universitario del Caribe, los 


\section{Calidad de vida en pacientes obesos sometidos a sleeve gástrico laparoscópico}

cuales fueron operados por el cirujano Arturo Hernández (desde el año 2012 hasta el II periodo del 2015) que residan en la ciudad de Cartagena de indias; tomando como población total 214 pacientes; Se realizó búsqueda y revisión de las historias clínicas.

A partir de los datos de filiación presentes en las historias clínicas, se obtuvo el número telefónico. Se realizó llamada telefónica a los pacientes, se explicó los alcances de la investigación y se pidió autorización para visita domiciliaria. Aquellos pacientes que estuvieron de acuerdo fueron evaluados aplicándoles los tres instrumentos.

Serán incluidos en la investigación los pacientes mayores de 18 años, de cualquier condición económica, edad, raza, y de cualquier procedencia, con obesidad mórbida sometidos a SGL; pacientes con índice de masa corporal mayor de $40 \mathrm{Kg} / \mathrm{m}$ que residan en la ciudad de Cartagena; pacientes intervenidos quirúrgicamente por el doctor Arturo Hernández y acepten la aplicación de la evaluación score BAROS que incluye el Test Moorehead-Ardelt (calidad de vida).

Serán excluidos los pacientes con IMC menor de $35 \mathrm{Kg} / \mathrm{m}$, pacientes que no residan en la ciudad de Cartagena y pacientes menores de 18 años de edad.

\section{Resultados}

Durante la realización del proyecto la población estuvo constituida por 60 pacientes con obesidad mórbida sometidos a SGL intervenidos por el cirujano Arturo Hernández en el E.S.E Hospital Universitario del Caribe, de los cuales 11 fueron intervenidos en el 2012, 24 pertenecientes al 2013, 13 al 2014 y por ultimo 12 en el 2015, aceptando participar el total de la población de manera voluntaria para la ejecución del mismo.

Tabla 1: Población del estudio

\begin{tabular}{|c|c|c|c|}
\hline VARIABLE SOCIODEMOGRÁFICA & CATEGORÍAS & $\mathrm{N}$ & $\%$ \\
\hline \multirow{2}{*}{ SEXO } & Masculino & 16 & 26,7 \\
\hline & Femenino & 44 & 73,3 \\
\hline TOTAL & & 60 & 100 \\
\hline \multirow{3}{*}{ OCUPACION } & Ama de casa & 15 & 25,0 \\
\hline & Empleado & 25 & 41,7 \\
\hline & Independiente & 20 & 33,3 \\
\hline TOTAL & & 60 & 100 \\
\hline \multirow{5}{*}{ ESTRATO SOCIOECONOMICO } & Uno & 23 & 38,3 \\
\hline & Dos & 20 & 33,3 \\
\hline & Tres & 9 & 15,0 \\
\hline & Cuatro & 5 & 8,3 \\
\hline & Cinco & 3 & 5,0 \\
\hline TOTAL & & 60 & 100 \\
\hline \multirow{3}{*}{ NIVEL DE FORMACIÓN } & Primaria & 11 & 18,3 \\
\hline & Secundaria & 18 & 30,0 \\
\hline & Superior & 31 & 51,7 \\
\hline TOTAL & & 60 & 100 \\
\hline \multirow{2}{*}{ ESTADO CIVIL } & Casado & 17 & 28,3 \\
\hline & Soltero & 17 & 28,3 \\
\hline
\end{tabular}


Calidad de vida en pacientes obesos sometidos a sleeve gástrico laparoscópico

\begin{tabular}{|c|c|c|c|}
\hline \multirow{2}{*}{} & Unión libre & 22 & 36,7 \\
\cline { 2 - 4 } & Divorciado & 2 & 3,3 \\
\cline { 2 - 4 } & Viudo & 2 & 3,3 \\
\hline \multirow{2}{*}{ TOTAL } & & 60 & 100 \\
\hline
\end{tabular}

Fuente: Elaboración propia

En la tabla 1, se observa que demográficamente el sexo predominante fue el femenino 73,3\%; (n:44), en un 41,7\%; $(n=25)$ de la población estudiada son empleados, el 38,3\% $(n=23)$ del total de la población son de estrato socio económico 1 , en un $51,7 \%(\mathrm{n}=31)$ el nivel de formación que tuvo más incidencia fue el superior, estando el $36,7 \%(n=22)$ de la población en estado civil unión libre.

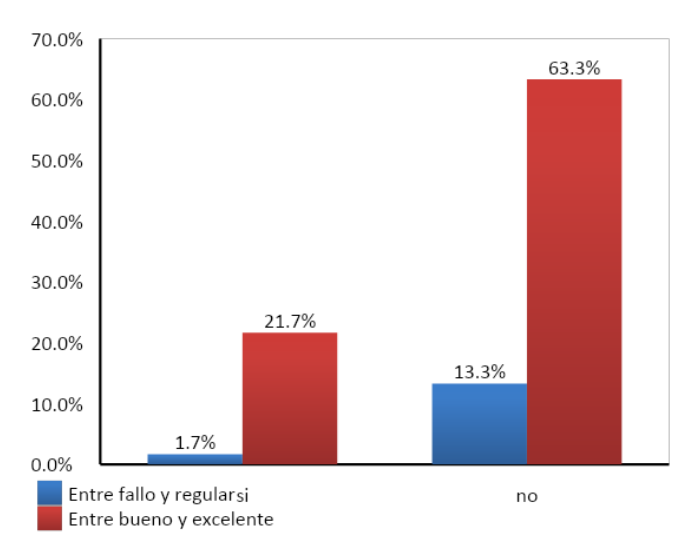

Gráfico 1: Calidad de vida según la presencia de dslipidemia (Elaboración propia)

Se evaluó un total de 60 casos, del total de estos se clasificaron con calidad de vida entre bueno y excelente a $51(85 \%)$ y entre fallo y regular a $9(15 \%)$.

Relacionando este hallazgo con la presencia de dislipidemia (Gráfico 1), se observó que 38 (63,3\%) de los $51(85 \%)$ casos con calidad de vida clasificada entre bueno y excelente no poseían este hallazgo, mientras que 13 (21.7\%) sí.

Este hallazgo puede conducir a plantear la asociación entre la presencia de Dislipidemia y calidad de vida. Para probar esta asociación se empleó una prueba de Chi cuadrado, estableciéndose como hipótesis nula (Ho) que no existe relación entre estas variables. Al aplicar la prueba se encontró un Chi cuadrado de 0.884 y una significancia 0.347 , por otra parte hubo un $25 \%$ de frecuencias esperadas menores a 5 . Lo cual conlleva a establecer que no existe suficiente evidencia estadística para rechazar la hipótesis nula y establecer la relación entre dislipidemia y calidad de vida. 


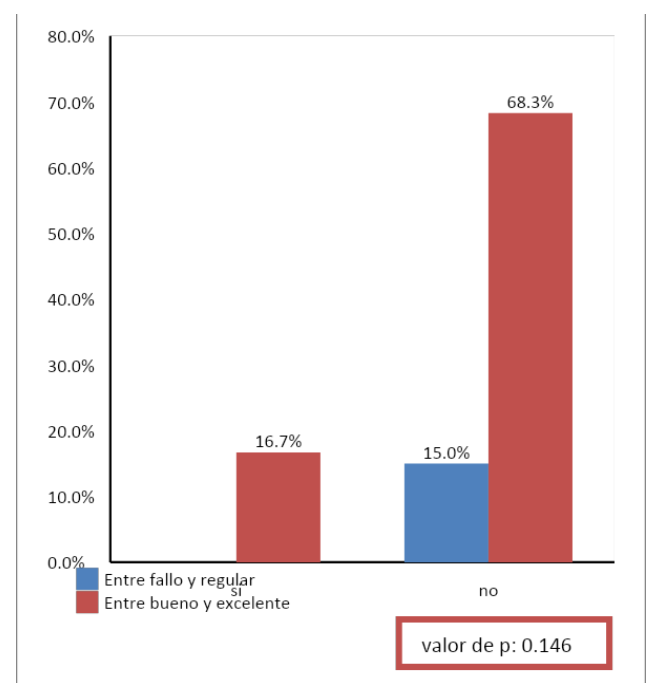

Gráfico 2: Calidad de vida según la presencia de diabetes (Elaboración propia)

Relacionando la presencia de Diabetes (Gráfico 2), se observó que 41 (68,3\%) de los 51(85\%) casos con calidad de vida clasificada entre bueno y excelente no poseían este hallazgo, mientras que $10(16,7 \%)$ si. Para probar esta asociación se empleó una prueba de Chi cuadrado, estableciéndose como hipótesis nula (Ho) que no existe relación entre estas variables. Al aplicar la prueba se encontró un Chi cuadrado de 2.1 y una significancia 0.146 por otra parte hubo un $25 \%$ de frecuencias esperadas menores a 5 . Lo cual conlleva a establecer que no existe suficiente evidencia estadística para rechazar la hipótesis nula y establecer la relación entre la presencia de Diabetes y calidad de vida.

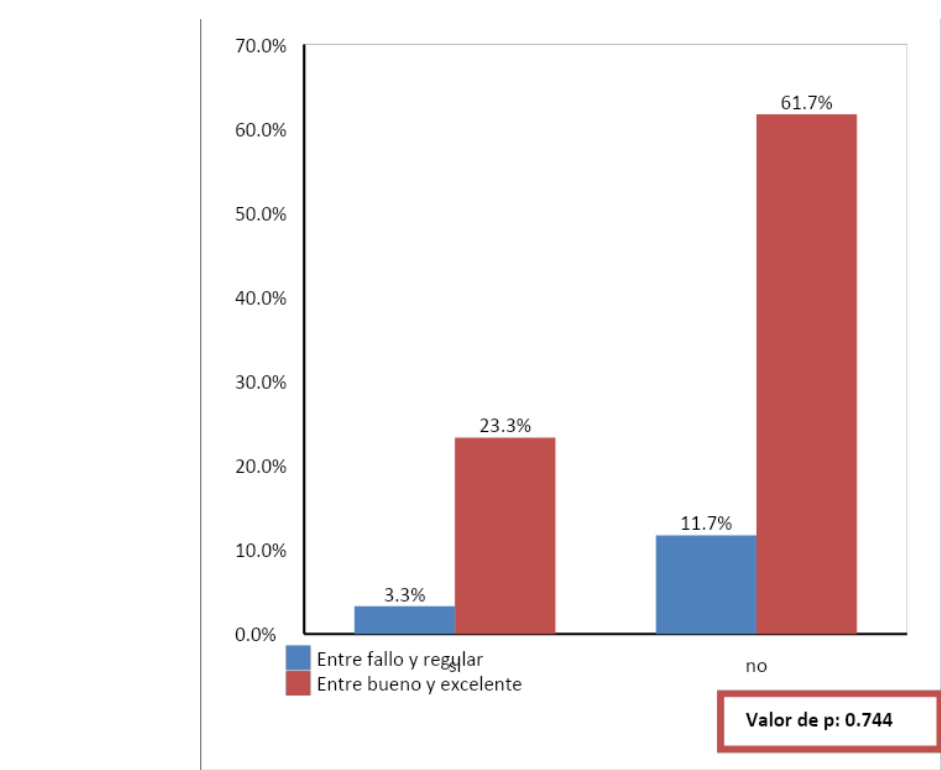

Gráfico 3: Calidad de vida según la hipertensión arterial (Elaboración propia)

En relación a la presencia de Hipertensión (Gráfico 3), en cuanto a calidad de vida buena excelente se encontró que de los pacientes con este calificativo en la calidad de vida el 23.3\% padecían hipertensión, mientras que el $61.7 \%$ no, pero al aplicar la prueba de significancia de chi cuadrado se halló un alfa de 0.744 y el $25 \%$ de las frecuencias esperadas asume que las dos variables guardan relación son menores de 5 , razón por la cual se infiere que no existe relación estadísticamente significativa entre estas dos variables. 


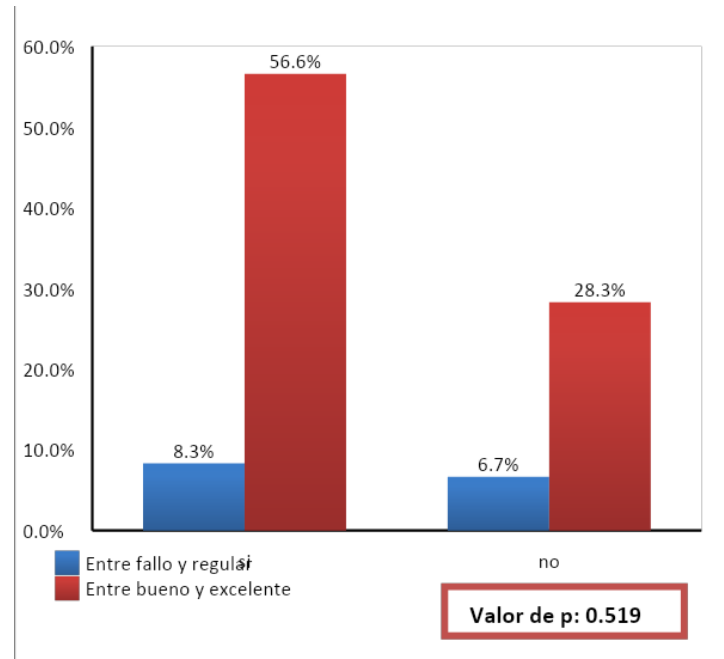

Gráfico 4: Calidad de vida según la presencia de afecciones osteomusculares (Elaboración propia)

Relacionando este hallazgo con la presencia de afecciones osteo-musculares (Gráfico 4) se observó que $34(56.6 \%)$ de los $51(85 \%)$ casos con calidad de vida clasificada entre bueno y excelente poseían este hallazgo, mientras que 17 (28.3\%) no. Para probar esta asociación se empleó una prueba de Chi cuadrado, estableciéndose como hipótesis nula (Ho) que no existe relación entre estas variables. Al aplicar la prueba se encontró un Chi cuadrado de 0.415 y una significancia 0.519 por otra parte hubo un $25 \%$ de frecuencias esperadas menores a 5. Lo cual conlleva a establecer que no existe suficiente evidencia estadística para rechazar la hipótesis nula y establecer la relación entre afecciones osteo-musculares y calidad de vida.

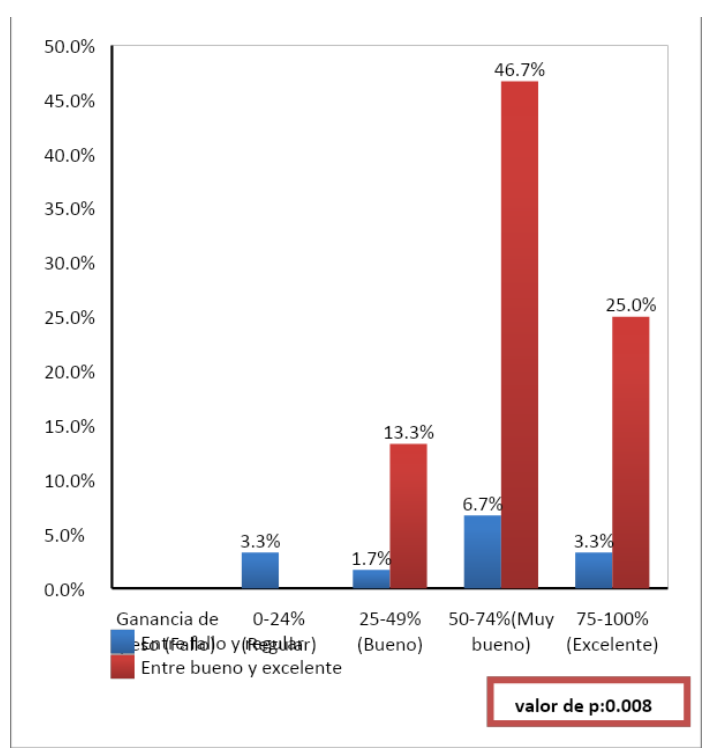

Gráfico 5: Calidad de vida según el porcentaje de sobrepeso perdido (Elaboración propia)

De aquí también se desprendió la evaluación de la calidad de vida según el porcentaje de peso perdido (Gráfico 5), teniendo así, que del 53\% de los pacientes que bajaron entre 50 y $74 \%$ el exceso de peso, solo IPSA SCIENTIA - Vol. $3 \mathrm{~N}^{\circ} 1-2018$ 


\section{Calidad de vida en pacientes obesos sometidos a sleeve gástrico laparoscópico}

el 46,7\% tienen una calidad de vida entre bueno y excelente; y del $28 \%$ que bajaron entre $75-100 \%$ el exceso de peso, solo el $25 \%$. Este hallazgo puede conducir a plantear la asociación entre el porcentaje de peso perdido y calidad de vida. Para este caso se comprobó una relación estadísticamente significativa, dado que el Chi cuadrado presenta una significancia de 0.008; por lo tanto se puede inferir que la mayor pérdida del porcentaje de sobrepeso perdido se relaciona con la buena calidad de vida en estos pacientes.

\section{Discusión}

El SGL es considerado el mejor tratamiento y más eficaz para la obesidad mórbida cuando la dieta junto con las modificaciones en el estilo de vida, el ejercicio, la terapia conductual y el tratamiento farmacológico han fallado (Mendes \& Vargas, 2017). Está técnica quirúrgica brinda como beneficio un descenso del exceso de peso y por ende una disminución en el IMC, grado de obesidad y una mejoría e incluso desaparición de las comorbilidades, lo que conlleva a un aumento de la autoestima, actividad física, laboral, social y sexual, mejorando la calidad de vida de estos pacientes.

Este estudio afirma los datos anteriores, ya que nuestros pacientes tras haber realizado innumerables dietas, ejercicio físico e incluso haber tomado medicamentos para la obesidad, no llegaron a perder el peso deseado. Sin embargo, a través de la cirugía se logró esta pérdida de peso, llegando a desaparecer o disminuir las comorbilidades y mejorando la calidad de vida de estos. Se ha encontrado similitud entre los resultados de esta investigación y los de numerosos estudios realizados.

Con relación a las comorbilidades, se evidencio una mejoría en la mayoría de los casos, inclusive la desaparición de algunas; transcurrido un tiempo después de la cirugía. Caso parecido al estudio de Alcaraz García et al., que arrojó datos semejantes a los resultados de esta investigación, mostro que han mejorado en casi todos los casos incluso desaparecido en algunos desde el primer año postquirúrgico (Alcaraz García et al., 2015; Bacallao et al., 2018).

Además se reflejó que a través de la técnica de SGL en la mayoría de los casos (53\%) presento un descenso del exceso de peso entre 50 y $74 \%$, representando esto una buena calidad de vida (EsquiviasZavala et al., 2016), ya que produce cambios significativos en las dimensiones bio-psico-social de la población(ver gráficos 4 y 5); Lo contrario de los resultados de otros estudios donde se evidencia que a través de la técnica de Banda Gástrica la mayoría de los casos (42\%) tuvo una pérdida del sobrepeso entre el 25 y 49\% demostrando de esta manera que la técnica quirúrgica SGL es la mejor opción para lograr el peso ideal (del Rosal et al., 2009; Aldaz et al., 2017).

Respecto al Score BAROS, el $90 \%$ de los pacientes presentaron una calidad de vida entre buena y excelente, debido que los aspectos de autoestima, actividad física, social, laboral y sexual mejoraron de manera considerable y notoria, esto mismos sucedió con el estudio de Alcaraz García et al., que tras un año de cirugía los resultados muestran que tanto la autoestima, como la actividad física, la actividad social, la actividad laboral, la actividad sexual y el comportamiento con la comida han mejorado considerablemente; por lo tanto la calidad de vida se encuentra entre buena y excelente para todos ellos (Alcaraz García et al., 2015; Fernández López, 2017).

Por último, en cuanto a la asociación de las variables con la calidad de vida demostró que el porcentaje de sobrepeso perdido y la actividad física tuvieron asociación mostrando un valor p: 0.008 y 0.017 


\section{Calidad de vida en pacientes obesos sometidos a sleeve gástrico laparoscópico}

respectivamente, similar al estudio de Velásquez-Zambrano et al., donde se demuestra la asociación de esta variables con la calidad de vida arrojando un valor p: 0.0144 y 0.0001 respectivamente. De esta manera se demuestra que el SGL repercute de manera positiva en la vida de este tipo de pacientes (del Rosal et al., 2009, Velásquez-Zambrano et al., 2014; Mejía \& Mpnro1, 2017).

Con relación a la actividad laboral y sexual no hubo asociación con la calidad de vida evidenciando un valor p: 0.098 y 0.247 respectivamente, lo mismo sucedió en el estudio de Velásquez-Zambrano et al., donde no hubo asociación de estos aspectos con la calidad de vida mostrando un valor p: 0.0624 y 0.6751 respectivamente. Se cree que hubo limitación por parte de la población al hablar ampliamente sobre su desempeño sexual con personas extrañas (del Rosal et al., 2009; Velásquez-Zambrano et al., 2014).

En cuanto a la autoestima se evidencia una asociación con la calidad de vida teniendo como valor p: 0.028. Lo contrario del estudio de Velásquez-Zambrano et al. (2014) donde esta no tuvo asociación con la calidad de vida obteniendo un valor p: 0.1145 . Con esto demostramos que este procedimiento quirúrgico (SGL) impacta de manera positiva en la perspectiva del individuo (Makarem-Kanso et al., 2008; García-Díaz et al., 2016).

\section{Conclusiones}

Teniendo en cuenta los objetivos de esta investigación y el análisis de los datos obtenidos mediante la aplicación del instrumento, se determinó que el SGL es el tratamiento de elección más efectivo para obtener una mejor calidad de vida luego de que otras medidas terapéutica hayan fallado. La pérdida de sobrepeso y la mejora de las comorbilidades influyo de manera positiva en el $90 \%$ de la población, conllevando a esta a obtener una calidad de vida entre buena y excelente. Así mismo Poseer un autoestima alto y realizar actividad física, conlleva a tener una óptima calidad de vida y sumado a esto, adquirir hábitos alimenticios saludables y llevar un seguimiento médico especializado favorecen al individuo a mantenerse como ser social y productivo.

\section{Referencias}

Acosta, V., Medina, R., Leyba, J. L., \& Navarrete, S. (2010). Calidad de vida en pacientes obesos sometidos a bypass gástrico laparoscópico en" y" de roux y gastrectomía vertical laparoscópica. Revista de la Facultad de Medicina, 33(1), 29-35.

Alcaraz García, A. M., Ferrer Márquez, M., \& Parrón Carreño, T. (2015). Calidad de vida en los pacientes obesos y su cambio tras cirugía bariátrica a medio y largo plazo. Nutrición hospitalaria, 31(5), 2033-2046.

Aldaz, R. Q., Matute, D. P., Atiencia, M., Caiche, R. Z., \& Quisiguiña, S. C. (2017). Gastrectomía vertical en manga laparoscópica y complicaciones tempranas. Debe reforzarse la línea de sutura?. Revista Médica-Científica CAMbios HECAM, 16(2), 30-34.

Argüello, A. B. A., \& Zúñiga, A. V. (2016). Cirugía bariátrica: generalidades. Medicina Legal de Costa Rica, 33(1), 145-153.

Bacallao, Y. G., Ríos, R. O., Ugarte, L. M., Piña, F. R., Zapata, Y. C., \& Fleitas, K. P. (2018). Calidad de vida relacionada con la salud en pacientes con obesidad. Revista Cubana de Medicina Física y Rehabilitación, 10(1), 48-60.

Basfi-Fer, K., Rojas, P., Carrasco, F., Valencia, A., Inostroza, J., Codoceo, J., ... \& Rojas, J. (2012). Evolución de la ingesta y del estado nutricional de zinc, hierro y cobre en mujeres sometidas a cirugía bariátrica hasta el segundo año postoperatorio. Nutrición Hospitalaria, 27(5), 1527-1535. 


\section{Calidad de vida en pacientes obesos sometidos a sleeve gástrico laparoscópico}

del Rosal, G., Scotti, N., Gullerian, A., \& Venini, C. (2009). Análisis de los cambios generados por la cirugía bariátrica en obesos mórbidos (tesis de grado). Buenos Aires, Argentina: Instituto Universitario de Ciencias de la Salud

Esquivias-Zavala, H., Reséndiz-Barragán, A. M., García, F., \& Elías-López, D. (2016). La salud mental en el paciente con obesidad en protocolo para cirugía bariátrica. Salud mental, 39(3), 165-173.

Fernández López, A. J. (2017). Eficacia terapéutica del bypass gástrico laparoscópico frente a la gastrectomía vertical laparoscópica al año de la cirugía bariátrica: factores de riesgo de fracaso de la cirugía y modelo predictivo de pérdida de peso inadecuada al año de la cirugía. Proyecto de investigación:.

García-Díaz, J. J., Ferrer-Márquez, M., Moreno-Serrano, A., Barreto-Rios, R., Alarcón-Rodríguez, R., \& Ferrer-Ayza, M. (2016). Resultados, controversias, y volumen gástrico después de la gastrectomía vertical laparoscópica en el tratamiento de la obesidad. Cirugía y Cirujanos, 84(5), 369-375.

Herrán Villalba, V., Lugo Calderón, E., Puello Martínez, A., \& Ruiz Restrepo, A. (2016). Nivel de conocimiento en riesgos laborales: el caso de trabajadores de servicios generales en una universidad. IPSA SCIENTIA, 1(1), 43-51.

Makarem-Kanso, Z., Miquilarena, R., Navas, H., Fermín, D. \& Jiménez, R. (2008). Manga Gástrica Laparoscópica, técnica para el tratamiento quirúrgico de la obesidad mórbida: experiencia en 70 casos. Revista Venezolana de Cirugía, 61(3), 125-130.

Marrugo Ligardo, Y. (2017). Health and safety management system in Latin America: a review from the hseq integration. IPSA SCIENTIA, 2(1), 38-45.

Mejía, C. R., \& Monroy, O. P. (2017). Fístula postmanga gástrica laparoscópica. Cirujano general, 38(3), 158-161.

Mendes, GA \& Vargas, GP (2017). Calidad de vida tras gastrectomía vertical evaluada mediante el cuestionario BAROS. A B C D. Arquivos Brasileiros de Cirurgia Digestiva (São Paulo), 30 (1), 14-17.

Pereferrer, F. S., Lanuza, E. D. A., Ibarzabal, A., Macias, M. S., Azcárate, V. V., de Gordejuela, A. G. R., ... \& Santos, R. S. (2017). Criterios de calidad en cirugía bariátrica: revisión de conjunto y recomendaciones de la Asociación Española de Cirujanos y de la Sociedad Española de Cirugía de la Obesidad. Cirugía Española, 95(1), 4-16.

Velásquez-Zambrano, J. G., Miranda-Fontalvo, A., Pulgar-Emiliani, M. I., Araujo-Zarate, P. L., \& Salazar-Mestre, C. J. (2014). Evaluación de la calidad de vida en pacientes obesos y cirugía bariátrica. Revista Ciencias Biomédicas, 5(1), 79-87.

Villaba Vimos, V., \& Vargas Ortiz, L. (2017). Work absenteeism in workers of an agrochemical manufacturing plant. IPSA SCIENTIA. Revista Científica Multidisciplinaria, 2(1), 10-20. 